\title{
Dielectric Materials, Design and Realization
}

\author{
Dorina M. Opris*a, Jose Enrico Q. Quinsaata, ${ }^{\mathrm{a}, \mathrm{b}}$, Simon Dünki ${ }^{\mathrm{a}, \mathrm{b}}$, Yee Song Ko ${ }^{\mathrm{a}, \mathrm{b}}$, Mihaela \\ Alexandru $^{\mathrm{a}}$, Carmen Racles ${ }^{\mathrm{c}}$, Frank A. Nüesch ${ }^{\mathrm{a}, \mathrm{b}}$, \\ a'Laboratory for Functional Polymers, Swiss Federal Laboratories of Materials Science and \\ Technology, Empa, Überlandstrasse 129, 8600 Dübendorf, Switzerland; ' Institute des Matériaux, \\ Ecole Polytechnique Fédérale de Lausanne, EPFL, Station 12, CH-1015 Lausanne; 'Petru Poni \\ Institute of Macromolecular Chemistry, Iasi, Romania.
}

\begin{abstract}
It has been the dream of many scientists to create polymeric materials which exhibit simultaneously high dielectric permittivity, low glass transition temperature, and excellent elastic properties. Such materials would be a highly attractive dielectric in electromechanical transducers. Within this topic we are focusing on silicones because of their excellent elastic properties over wide temperature and frequency ranges combined with low glass transition temperatures. To increase their low permittivity, we followed different approaches which include: blending the matrix with highly polarizable conductive and polar nanofillers and chemical modification with polar side groups. This presentation will show the advantages and disadvantages of the two strategies we have been following and will provide an assessment of their future potential.
\end{abstract}

Keywords: dielectric elastomer actuators, silicone, functionalized silicones, high permittivity

\section{INTRODUCTION}

High dielectric permittivity $\left(\varepsilon^{\prime}\right)$ elastomers are attractive materials for dielectric elastomer transducers (DET). ${ }^{1}$ DET are stretchable devices constructed from an elastic films coated with two compliant and stretchable electrodes. ${ }^{2}$ They can be used as actuators, generators, and sensors. Irrespective of the final application, elastomers with increased $\varepsilon^{\prime}$ are attractive since they operate at lower voltage. ${ }^{3}$ Therefore, the access to high $\varepsilon^{\prime}$ elastomers with low conductivity, high breakdown strength, high strain at break, and low viscoelastic losses might increase the commercial potential of DET. ${ }^{4}$

Elastomers undergo reversible deformation under an external load, but they tend to have an $\varepsilon^{\prime}$ below $\varepsilon^{\prime}=3$. Many elastomers like polyisoprene, polyurethane, polyalkylacrylates, polyacrylonitrile-polybutadiene, natural rubber, and polysiloxanes have already been tested in DET. From all polymers, polysiloxanes are often used. ${ }^{5}$ They have a Si-O backbone in which the silicone atoms typically carry two organic hydrocarbon substituents. Polydimethylsiloxane (PDMS) has two methyl groups and a low glass transition temperature of about $-120^{\circ} \mathrm{C}$, a melting temperature of $-40^{\circ} \mathrm{C}$, excellent thermal properties, and resistance to oxidation. ${ }^{5}$ Since PDMS is a viscous liquid, it has to be cross-linked to a free standing film. For cross-linking, either functional or non-functional PDMS can be used. When non-functional PDMS are used, their chains can be randomly connected by using the peroxide or e-beam methods. ${ }^{5}$ The mechanical properties are tuned by using different amount of peroxides or different irradiation times. Appropriate end-functionalized PDMS can also be cross-linked. For example, vinyl end-functionalized PDMS are cross-linked via an addition cure reaction of hydrosilyl groups containing cross-linker in the presence of a platinum catalyst. ${ }^{6}$ Vinyl end-functionalized PDMS can also be cross-linked via a thiol-ene addition for which a multifunctional cross-linker is used. ${ }^{7}$ While the hydrosilylation is sensitive to impurities (which can hinder the cross-linking), the thiol-ene reaction is rather fast and easy to perform.

Hydroxyl end-functionalized PDMS have also been used in a condensation cure to form silicone networks. Depending on the type of cross-linker used, the reaction rate can be influenced. ${ }^{8}$ Typical cross-linkers used are tetraethoxysilane, oligosiloxane containing hydrosilyl groups, or tetrakis(dimethylsiloxy)silane together with dibutyltin dilaurate catalyst or ethyltriacetoxysilane together with titanium 2-ethylhexoside catalyst. The advantage of this system rests in the reliability of this reaction, which is practically insensitive to impurities and fillers. This condensation cure reaction was therefore intensively exploited in our laboratories. ${ }^{9}$

Electroactive Polymer Actuators and Devices (EAPAD) 2015, edited by Yoseph Bar-Cohen, Proc. of SPIE Vol. 9430, 94300A · C 2015 SPIE · CCC code: 0277-786X/15/\$18 - doi: 10.1117/12.2086134 
Unfortunately, irrespective of the cross-linking reaction used for the formation of the network, elastomers with rather low $\varepsilon^{\prime}$ are formed. Several approaches have been described in the literature to increase the $\varepsilon^{\prime}$ of a polymer by blending it with high $\varepsilon^{\prime}$ fillers like ceramic or conductive particles, or by chemical modification of the polymer with polar side groups. ${ }^{1}$ However, in many cases, an increase in $\varepsilon^{\prime}$ was reported, while other important material properties were negatively affected. It is therefore still a great challenge to synthesize elastomers that have a combination of properties like: high $\varepsilon$ ', low dielectric losses and conductivity, low $Y$ with large strain at break, low viscoelastic losses as well as high dielectric breakdown. This presentation gives an overview of materials developed in our lab and shows the advantages and disadvantages of the strategies we have been following.

\section{RESULTS AND DISCUSSION}

\section{Silicone matrices}

As mentioned in the introduction silicone elastomers with good mechanical properties are known and some formulations are commercially available. However, most of the commercial formulations use a hydrosilylation reaction for crosslinking which is rather sensitive to impurities and added filler. In the worst case the cross-linking reaction is completely inhibited. Therefore, it was the aim of our work to develop reliable cross-linking reactions which allow formation of silicone elastomers with low viscoelasticity, low $Y$, high strain at break, and tuneable mechanical properties. Since the molecular weight $\left(M_{w}\right)$ of PDMS has a direct impact on the mechanical properties, a hydroxyl end-functionalized PDMS of a rather high molecular weight $\left(M_{w}=139 \mathrm{kDa}\right)$ was used as starting material. As cross-linkers, two different poly(dimethyl-methylhydro)siloxanes were used: a 25-35\% methylhydrosiloxane-dimethylsiloxane-copolymer $\left(M_{w}=2\right.$ $\mathrm{kDa}$ ) for series A and Neukasil A7 for series B (Scheme 1). ${ }^{9}$ In a typical preparation the hydroxyl end-functionalized PDMS, the cross-linker and the catalyst (dibutyltindilaurate) were mixed in different proportions as shown in Table 1 and the viscosity of the mixture was adjusted with toluene. The thin films made by the doctor blade technique were allowed to cross-link at room temperature. The samples were aged for several weeks before testing. Silicone materials with similar dielectric properties but rather different mechanical properties were obtained.

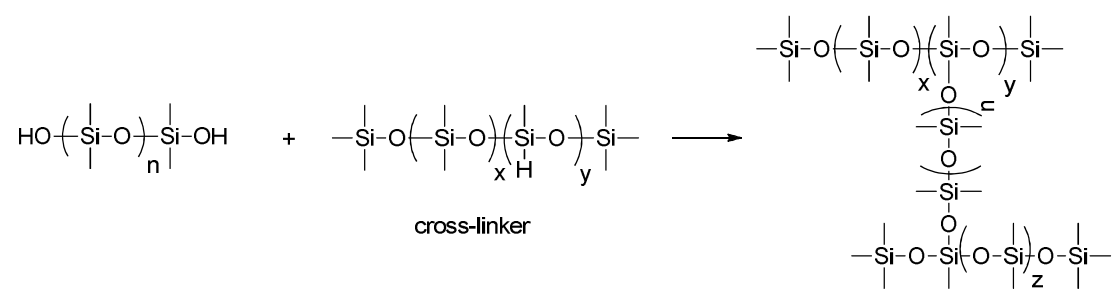

Scheme 1. The cross-linking reaction used for the formation of room temperature cross-linked silicones.

Table 1. The amount of reagents used for the synthesis of different silicones as well as the elastic modulus at $10 \%$ strain $\left(Y_{10 \%}\right)$, the lateral actuation strain at $30 \mathrm{~V} / \mu \mathrm{m}\left(s_{l}\right)$, maximum lateral actuation strain $\left(s_{l}(\max )\right)$ at breakdown $\left(E_{b}\right)$.

\begin{tabular}{cccccccc}
\hline Entrance & $\begin{array}{c}\text { PDMS } \\
{[\mathrm{g}]}\end{array}$ & $\begin{array}{c}\text { CL } \\
{[\mathrm{ml}]}\end{array}$ & $\begin{array}{c}\text { Catalyst } \\
{[\mu \mathrm{l}]^{\mathrm{a}}}\end{array}$ & $\begin{array}{c}Y_{10 \%}{ }^{\mathrm{d}} \\
{[\mathrm{kPa}]}\end{array}$ & $\begin{array}{c}s_{l} \text { at } 30 \mathrm{~V} / \mu \mathrm{m} \\
{[\%]}\end{array}$ & $\begin{array}{c}s_{l}(\max ) \\
{[\%]}\end{array}$ & $\begin{array}{c}E_{b}{ }^{\mathrm{e}} \\
{[\mathrm{V} / \mu \mathrm{m}]}\end{array}$ \\
\hline $\mathbf{A 1}$ & 5 & $0.8^{\mathrm{b}}$ & 25 & 440 & 1.8 & 13.2 & 62.2 \\
$\mathbf{A 2}$ & 5 & $0.2^{\mathrm{b}}$ & 25 & 540 & 1.3 & 10.9 & 72.3 \\
B1 & 5 & $3^{\mathrm{c}}$ & 25 & 210 & 5.4 & 12.4 & 40 \\
B2 & 5 & $5^{\mathrm{c}}$ & 25 & 130 & 10.8 & 13.9 & 32 \\
\hline
\end{tabular}

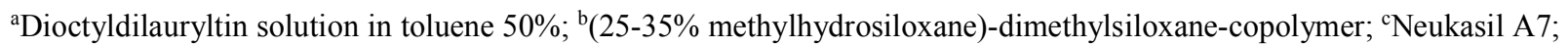
${ }^{\mathrm{d}} Y$ was determined from the slope of the stress-strain curves using a linear fit to the data points within $10 \%$ strain; ${ }^{\mathrm{e}} E_{\mathrm{b}}$ from actuator measurements.

Figure 1 shows the stress-strain curves obtained from the tensile tests of the four materials prepared. All samples withstood strains of at least $150 \%$. Materials of series A are stiffer than materials of series $\mathbf{B}$. The mechanical properties within a series can be tuned by the amount of cross-linker used. 


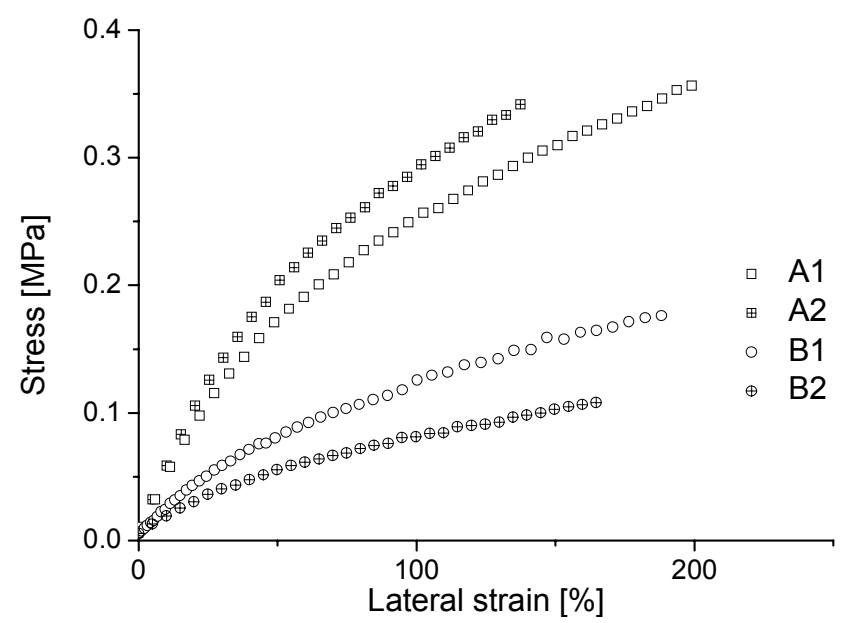

Figure 1. Stress-strain curves of the different silicone materials prepared. The stress-strain curves were averaged from at least four independent tests and the minimum strain at break for each material is shown.

\section{High permittivity silicone based materials}

Silicone based materials with increased permittivity were prepared by following two approaches: blending high $\varepsilon^{\prime}$ fillers in silicone matrices with good mechanical properties or chemical modification of polysiloxane backbone with polar groups. The materials described below are named as $S_{x}^{y}$ where $\mathrm{S}$ represents the silicone matrix, $\mathrm{x}$ represents the type of filler or polar group used to increase $\varepsilon^{\prime}$ and y represents the vol\% of filler or mol\% of polar group used, respectively.

\section{Synthesis of conductive fillers and their composites in silicone}

Having in hand several silicones with good elastic properties, it was the second aim of our work to go one step further and to increase their $\varepsilon^{\prime}$.

It has been shown in the literature that conductive fillers can be used to increase the $\varepsilon^{\prime}$ of a matrix when the concentration of the filler approaches the percolation threshold. ${ }^{10}$ We explored two fillers: silver nanoparticles (AgNPs) and polyaniline. AgNPs were selected because despite their relatively high price, they can be prepared in defined sizes which are kept unchanged during processing. They therefore served as a good model system and allowed us to investigate how the dielectric properties are affected by this metallic filler. On the other hand, polyaniline is cheap, easy to prepare, and has a good environmental stability but its morphology is more difficult to control. When conductive fillers are used to increase the $\varepsilon^{\prime}$ of a material, special care has to be taken to avoid that conductive paths are formed through the material. To circumvent this issue, the conductive particles were equipped with an insulating shell prior to blending. This is a powerful means to avoid formation of conductive paths through the blended film even when particles agglomerate.

Sufficient amount of AgNPs were prepared using the polyol synthesis in a segmented flow tubular reactor. ${ }^{11}$ Particles with diameters on the order of $38 \mathrm{~nm}$ were produced. They were coated with silica shells of different thicknesses ranging from 3.6 to $20 \mathrm{~nm}\left(\mathrm{Ag} @ \mathrm{SiO}_{2}(\mathrm{x})\right.$; where $\mathrm{x}$ represents the thickness of the silica shell) (Figure 2). ${ }^{12}$ The dielectric properties of such particles were measured in pressed pellets in parallel-plate capacitors. Figure 3 shows the $\varepsilon$ ' as function of frequency for particles having silica shells of different thicknesses. The dielectric properties of $\mathrm{Ag}_{0} \mathrm{SiO}{ }_{2}(20)$ are similar to those of neat silica, indicating that the Ag core is effectively decoupled from the environment by the silica shell. $\mathrm{Ag} @ \mathrm{SiO}_{2}(6.6)$ particles have $\varepsilon^{\prime} \sim 40$ at high frequencies, while $\mathrm{Ag} @ \mathrm{SiO}_{2}(<4)$ particles were so conductive that dielectric measurements could not be performed. It was therefore concluded that the optimal silica shell thickness should be around $4 \mathrm{~nm}$ to allow a reasonable increase in permittivity. 

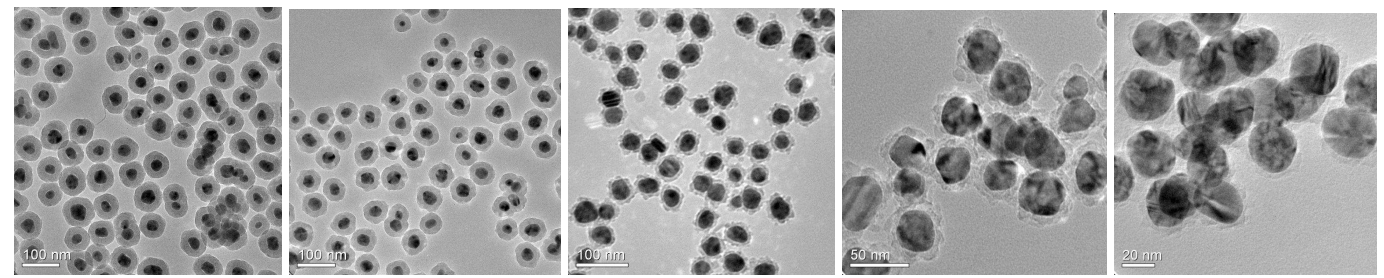

Figure 2. TEM images of the AgNPs coated with a silica shell. The average diameters of AgNPs were $38 \mathrm{~nm}$ while the $\mathrm{SiO}_{2}$ shells were about $20 \pm 2 \mathrm{~nm} ; 17 \pm 2 \mathrm{~nm} ; 8 \pm 1.5 \mathrm{~nm} ; 6.6 \pm 1.5 \mathrm{~nm} ; 3.6 \pm 1 \mathrm{~nm}$ from left to right.

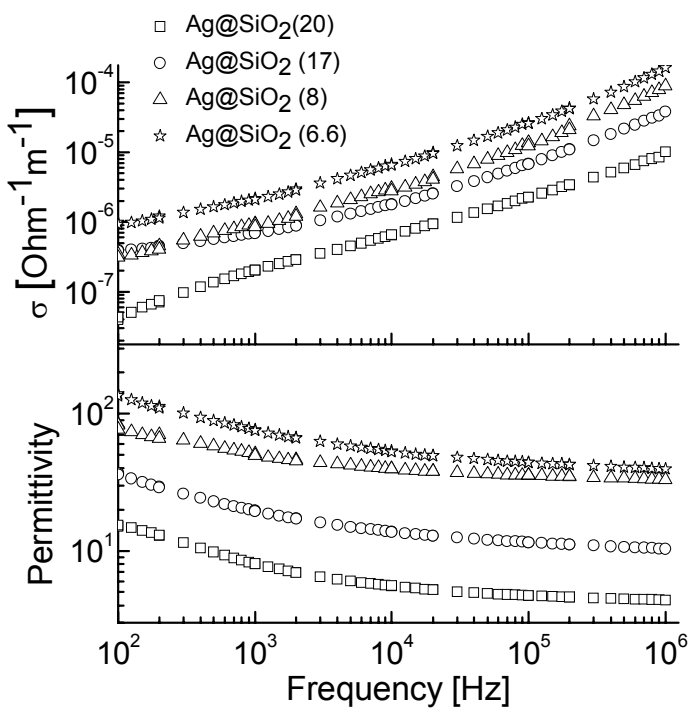

Figure 3. Permittivity and conductivity of AgNPs coated with silica shells of different thickness as function of frequency.

Next, we prepared AgNPs $(\sim 57 \mathrm{~nm})$ on a large scale and coated them with a thin silica shell $(<4 \mathrm{~nm})$ functionalized with hexamethyldisilazane. This surface functionalization allowed dispersion of these particles in non-polar solvents. TEM images of the $\mathrm{Ag} @ \mathrm{SiO}_{2}(<4)$ particles used are shown in Figure 4. As can be seen, the silica shells are rather homogeneous and of constant thickness reflecting controlled coating procedure. The dielectric properties of those particles were measured in pellets and found to have an almost frequency independent values of $\varepsilon^{\prime}=90$ and a very low loss factor $\tan \delta=0.023$ at high frequencies.

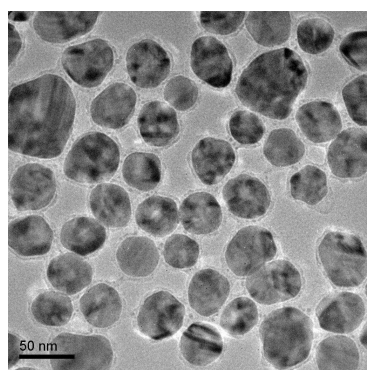

Figure 4. TEM images of the AgNPs coated with a silica shell and subsequently functionalized with hexamethyldisilazane. The average diameter of AgNPs was $54 \mathrm{~nm}$ while the $\mathrm{SiO}_{2}$ shells were slightly below $4 \mathrm{~nm}$. A strong contrast between the black Ag core and the grey silica shell can be seen. 
The second filler, PANI, was obtained by encapsulating polyaniline with cross-linked polydivinylbenzene (PDVB) which could easily and homogenously be achieved using a miniemulsion polymerization protocol of divinylbenzene ${ }^{8}$ By SEM it was shown that nanoparticles indeed form (Figure 5). The elemental analysis shows that about $13 \%$ polyaniline was encapsulated. The position of polyaniline in the particles was determined by comparing the $\mathrm{C} / \mathrm{N}$ ratio from the elemental analysis with the $\mathrm{C} / \mathrm{N}$ ratio of the surface of the particles by X-ray photoelectron spectroscopy (XPS). The smaller $\mathrm{C} / \mathrm{N}$ ratio from the elemental analysis as compared to the one from XPS indicates that the polyaniline is covered by the PDVB. The encapsulation process was successfully scaled up such that PANI particles on $100 \mathrm{~g}$ scale can be prepared.

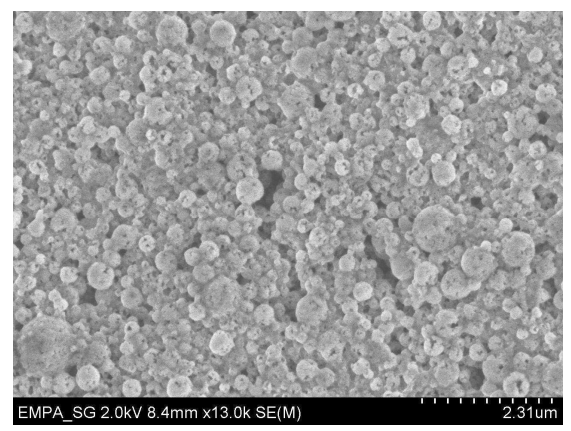

Figure 5. SEM micrographs of the PDVB/PANI particles.

Having access to the above described fillers, silicone composites were made. Materials containing different vol\% of fillers were prepared and their dielectric and mechanical properties were investigated. In all cases, an increase in the $\varepsilon$ ' and an improvement in the strain at break was observed with increasing vol\% of filler (not shown) up to 20 vol\%. Material $S_{P A N I}^{25}$ has $\varepsilon^{\prime}=4.0$, a strain at break as high as $1400 \%$ and an elastic modulus of $Y_{10 \%}=34.5 \mathrm{kPa}$ at $10 \%$ strain but shows some viscoelastic losses. A further increase of PANI vol\% does not allow formation of materials with good elastic properties. ${ }^{9}$ Material $S_{A g}^{20}$ shows a $\varepsilon^{\prime}=5.9$ at $\sim 1 \mathrm{kHz}$, a dielectric strength of $13.4 \mathrm{~V} / \mu \mathrm{m}$, elastic modulus of $Y_{10 \%}=$ $2.3 \mathrm{MPa}$ and $Y_{100 \%}=350 \mathrm{kPa}$, and a strain at break of $800 \%$. By increasing the AgNPs vol\% to $30 \%$, a flexible material with $\varepsilon^{\prime}=21$ and a loss factor of 0.03 at $1 \mathrm{kHz}$ formed, but the elastic properties as well as the breakdown strength were strongly deteriorated. ${ }^{13}$ Figure 6 shows the stress-strain curves of selected materials $S_{P A N I}^{20}, S_{P A N I}^{25}$ and $S_{A g}^{20}$ and includes further materials that will be discussed below. An overview of the electromechanical properties of these materials is given in Table 2 .
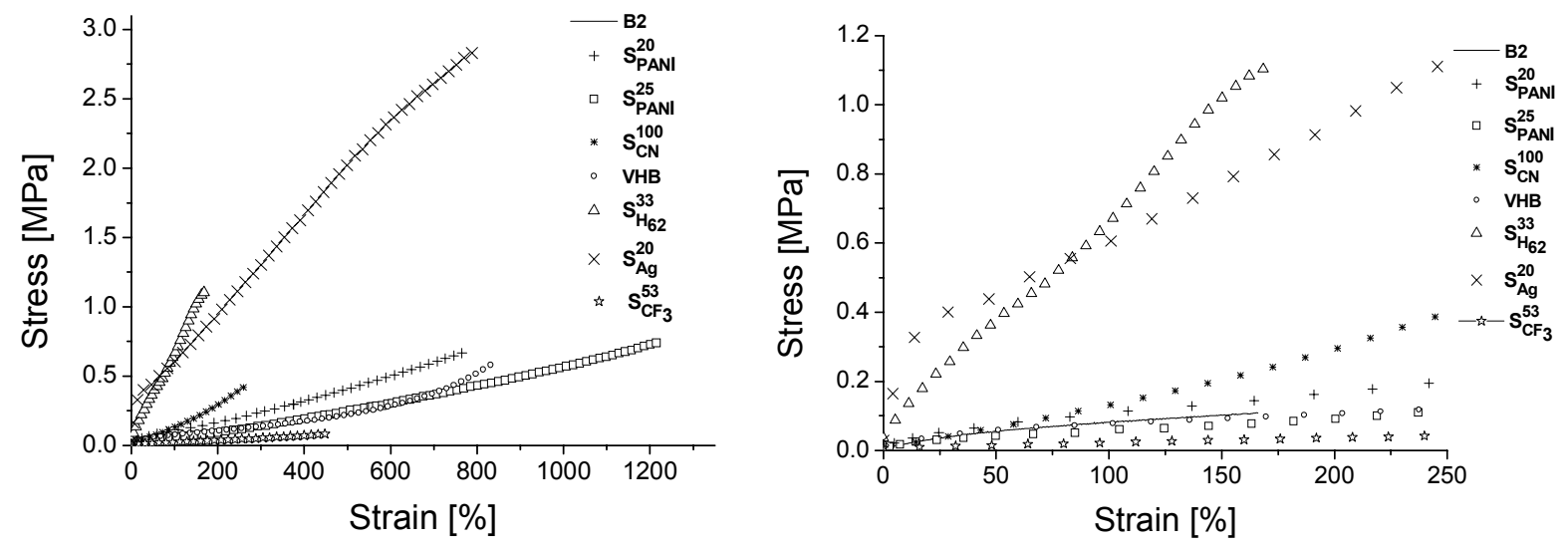

Figure 6. Stress-strain curves of different silicone materials (left) with the low strain regime enlarged (right). The stressstrain curves were averaged from at least three independent tests and the minimum strain at break for each material was taken. 


\section{Synthesis of new polar silicones}

\section{Silicones containing polar nitrile groups}

Two different series of polysiloxane containing polar nitrile groups were prepared $(\mathbf{H x}$ and $\mathbf{T} \mathbf{x}$, where $\mathrm{H}$ or $\mathrm{T}$ represents series name and $\mathrm{x}$ represents the mol\% of polar nitrile groups) (Scheme 2). Series Hx was prepared by co-hydrosilylation starting from trimethylsilyl end-terminated poly(methylhydro)siloxane and different allyl cyanide $/ \mathrm{n}$-hexene ratios. ${ }^{14}$ Series Tx was prepared starting from a polymethylvinylsiloxane 1 which was reacted with 1-butanethiol (2) and 3mercaptopropionitrile $\mathbf{3}$ both separately to give polymers $\mathbf{T} 2$ and $\mathbf{T 3}$, respectively, as well as in various ratios $\mathrm{x}, \mathrm{y}$ so as to create polymers $\mathbf{T} \mathbf{2}_{\mathbf{x}} \mathbf{T} 3_{\mathbf{y}}$ with various contents of the polar nitrile group (y). ${ }^{15}$ While the modifications of poly(methylhydro)siloxane did not proceed quantitatively even when excess of reagents and long reaction times were used, the thiol-ene modification of $\mathbf{1}$ was fast, reliable, and quantitative.

a)

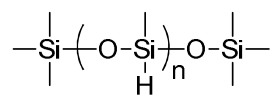

b)

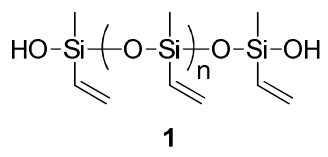

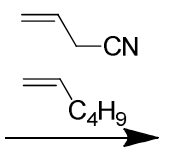

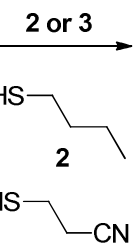

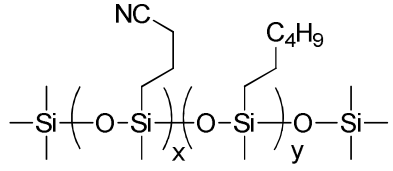

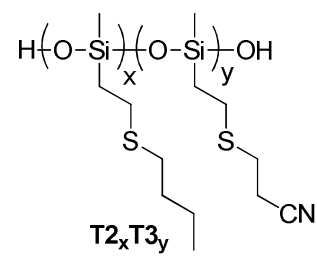

\begin{tabular}{|c|c|c|}
\hline T2: & & 1:0 \\
\hline T2, T3 & & \\
\hline $\mathrm{T} 2_{8} \mathrm{~T} 3_{2}$ & & \\
\hline $\mathrm{T} 2{ }_{7} \mathrm{~T} 3_{3}$ & & \\
\hline $\mathrm{T} 2_{6} \mathrm{T3}_{4}$ & & \\
\hline $\mathrm{T} 2_{1} \mathrm{~T} 3_{1}$ & & \\
\hline $\mathrm{T} 2_{4} \mathrm{~T} 3_{6}$ & & \\
\hline $\mathrm{T} 2_{3} \mathrm{~T} 3_{7}$ & & \\
\hline T3: & & \\
\hline
\end{tabular}

Scheme 2. Synthesis of series $\mathbf{H}$ by hydrosilylation of allyl cyanide/n-hexene with poly(methylhydro)siloxane (a) and of series $\mathbf{T}$ by thiol-ene addition of 1-butanethiol/3-mercaptopropionitrile to polymethylvinylsiloxane (b).

The dielectric properties of polymers containing different amount of polar nitrile groups were investigated at different frequencies. Irrespective of the type of reaction used for the introduction of the nitrile groups, a linear increase in the $\varepsilon^{\prime}$ with increasing the content of nitrile groups was observed. For example, series $\mathbf{T}$ exhibits permittivities ranging from $\varepsilon^{\prime}=$ 4.7 to 18.4 for $\mathbf{T} 2$ and $\mathbf{T 3}$, respectively (Figure 7). The $\varepsilon^{\prime}$ values of all polymers $\mathbf{T} \mathbf{2}_{\mathbf{x}} \mathbf{T} \mathbf{3}_{\mathbf{y}}$ lie within this range.

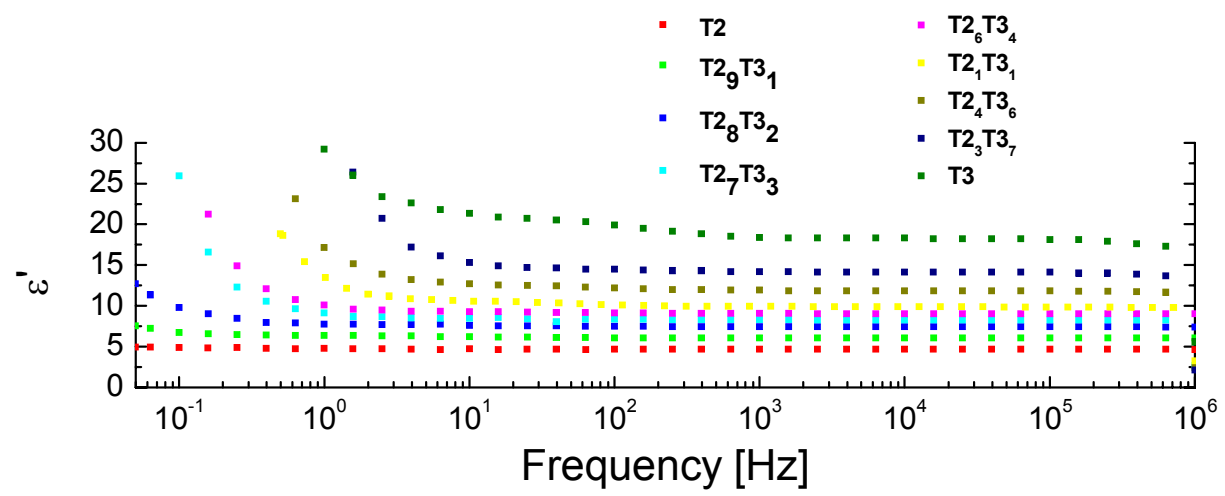

Figure 7. Dielectric permittivity $\left(\varepsilon^{\prime}\right)$ of $\mathbf{T} 2, \mathbf{T} 3$ and $\mathbf{T} \mathbf{2}_{\mathbf{x}} \mathbf{T} \mathbf{3}_{\mathbf{y}}$ at room temperature. 
Silicone elastomers modified with polar cyano groups

The polysiloxanes modified with nitrile groups can be used as high $\varepsilon^{\prime}$ fillers in a polymer matrix with good mechanical properties. The difficulties encountered here are the incorporation of a large amount of polar component while avoiding the macrophase separation of the two polymers of different polarity. Therefore we also explored the possibility of incorporating large amounts of silicones containing polar nitrile groups into a non-polar silicone matrix by using an interconnected network. A three steps process starting from hydroxyl end-functionalized polydimethylsiloxanes (PDMS) of high molecular weight $\left(M_{w}=139 \mathrm{kDa}\right)$, trimethylsilyl-end blocked silicones that carry hydrosilane, cyanopropyl and hexyl groups $\mathbf{H}_{62}$, and tetraethoxysilane (TEOS) was developed. The unreacted hydrosilane groups of $\mathbf{H}_{62}$ were first hydrolyzed. The formed hydroxyl groups were subsequently reacted with partially hydrolyzed TEOS and further used as high $\varepsilon^{\prime}$ component, cross-linker, and reinforcing agent for the PDMS matrix. Materials with increased permittivity formed. Herein material $S_{H_{62}}^{33}$ with an elastic modulus of $800 \mathrm{kPa}$ at $10 \%$ strain (Figure 6), $\varepsilon^{\prime}=4.5$, and a maximum actuation strain of $8 \%$ at $E_{b}=56 \mathrm{~V} / \mu \mathrm{m}$ was selected for further discussions.

The above described series T has hydroxyl end-groups which should allow for cross-linking. Preliminary experiments show that materials with good elastic properties form, however the amount of material prepared did not allow us a detailed investigation of their properties. Further work is invested in our group in this direction.

The functionalization of a poly(methylvinyl)siloxane is not only possible via a typical post-polymerization modification reaction in solution as the one described above, but also in thin films. Thus, a one-step synthesis in thin films starting from poly(methylvinyl)siloxane, 3-mercaptopropionitrile, and 2,2'-(ethylenedioxy)diethanethiol was developed. ${ }^{16}$ While 3-mercaptopropionitrile allows modification of the starting polymer 1 with polar nitrile groups, the 2,2'(ethylenedioxy)diethanethiol allows for cross-linking (Figure 8). This chemical modification provides us with material $S_{C N}^{100}$, with a powerful combination of properties: $\varepsilon^{\prime}=10.1$, elastic modulus $Y_{10 \%}=154 \mathrm{kPa}$, and strain at break of $260 \%$ (Figure 6).

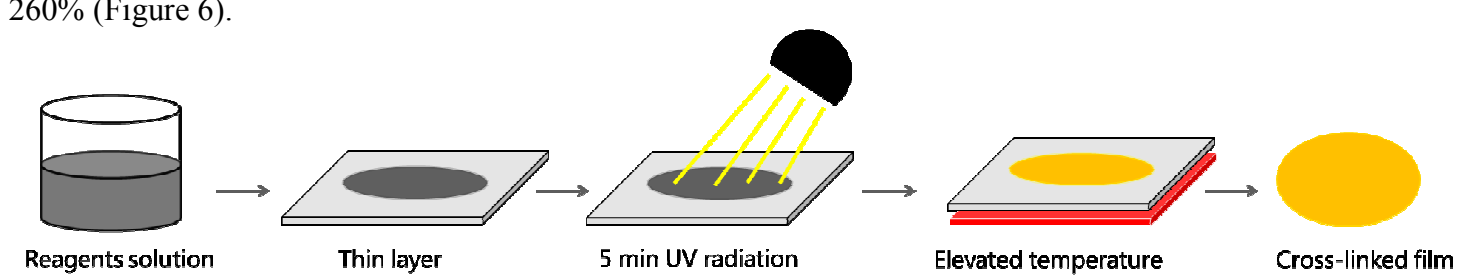

Figure 8. Synthesis of nitrile containing silicone elastomer $S_{C N}^{100}$ starting from a solution of reagents. A thin layer is first formed by doctor blade which is irradiated for $5 \mathrm{~min}$ with UV light. After drying the formed film at elevated temperatures, the film can be peeled off.

Silicone elastomers modified with polar trifluoropropyl groups

Another polar group investigated by us was the trifluoropropyl group. A vinyl-terminated polysiloxanes containing 53 mol\% trifluoropropyl groups was prepared starting from 1,3,5-tris(3,3,3-trifluoropropyl)-1,3,5-trimethylcyclotrisiloxane $\left(\mathrm{F}_{3}\right)$ and octamethylcyclotetrasiloxane $\left(\mathrm{D}_{4}\right)$ via anionic bulk polymerization. The cross-linking of this trifluoropropyl modified silicone using the vinyl end-groups and tetrakis(dimethylsiloxy)silane cross-linker via a hydrosilylation reaction allowed formation of material $\mathrm{S}_{\mathrm{CF}_{3}}^{53}$ with a permittivity $\varepsilon^{\prime}=6$ and a strain at break as high as 460\% (Figure 6).

\section{Electromechanical tests}

Several silicone based materials for actuator applications were developed in our labs. Herein, we also describe the electromechanical performance of some selected materials. Films from different materials were strained and fixed on a rigid frame. Circular electrodes $(8 \mathrm{~mm}$ diameter) of carbon black powder were applied to each side of the film. The voltage was increased by $100 \mathrm{~V}$ up to breakdown. The actuation strain was measured optically as the extension of the diameter of the electrode area via a digital camera. It should be mentioned here that the strain used for different materials was not exactly the same since some of materials did not withstand large strains.

The actuation strain in thickness direction is given by:

$$
s_{z}=p / Y=\frac{\varepsilon \varepsilon_{0}}{Y}\left(\frac{U}{d}\right)^{2}
$$


where $p$ is the electrostatic pressure acting on the film, $Y$ is the Young's modulus, $\varepsilon_{0}$ is the permittivity of free space and $\varepsilon$ is the dielectric permittivity of the polymer, $U$ is the applied voltage, and $d$ is the thickness of the film. Since the actuation strain of a material is influenced by the $\varepsilon^{\prime}$ and $Y$, Table 2 gives an overview of $\varepsilon^{\prime}$ and $Y$ at 10 and $100 \%$ strain, the $\varepsilon^{\prime} / Y$ values, as well as $E_{\mathrm{b}}$ and the maximum lateral actuation strain at $E_{b}$.

Since materials $\mathbf{A}$ and $\mathbf{B}$ have similar chemical composition, their dielectric permittivity is the same (about 2.5). Therefore the different performance of material $\mathbf{A}$ and $\mathbf{B}$ in the actuator test is due to the difference in their mechanical properties. The determined actuation strains are shown in Figure 9 as a function of electric field. The softest material $\mathbf{B}_{2}$ gave the larger actuation strain of $10.8 \%$ at $30 \mathrm{~V} / \mu \mathrm{m}$, while the stiffer material $\mathbf{A} 2$ gave an actuation strain of $1.3 \%$ at the same voltage. As expected, a linear increase in the actuation strain at the same voltage with decreasing the elastic modulus was observed. Additionally, the $E_{b}$ decreases with decreasing the elastic moduli.
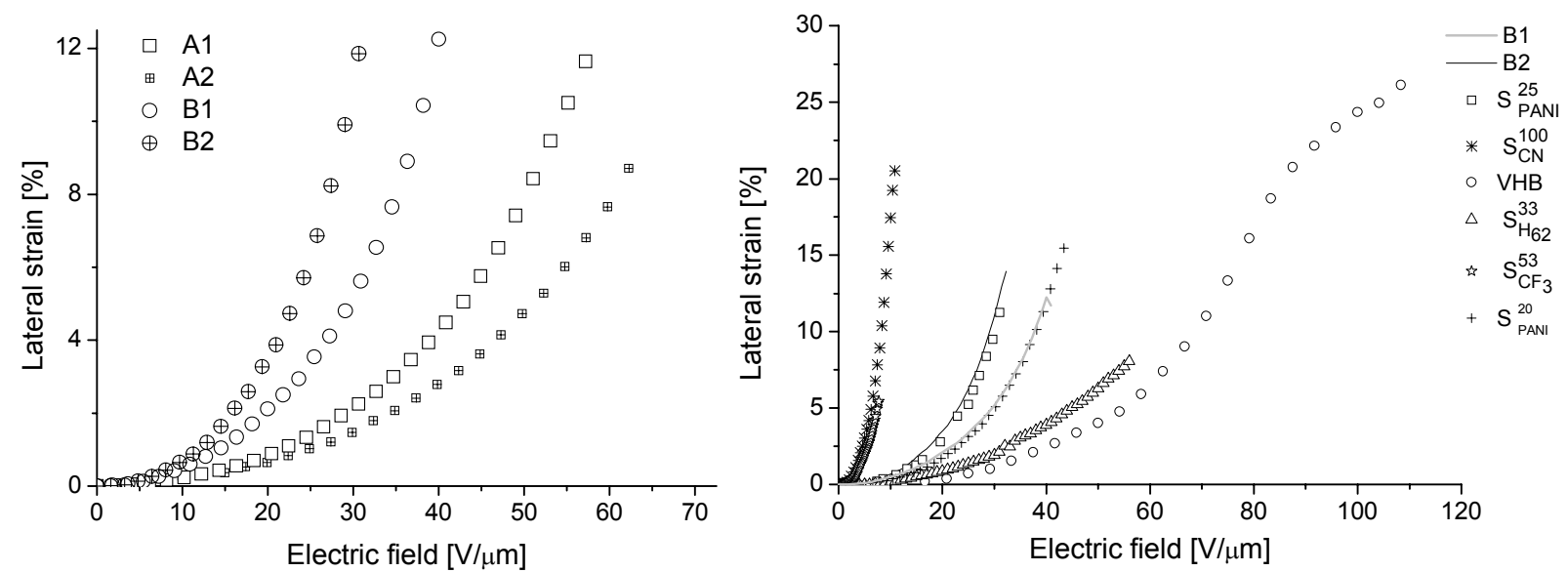

Figure 9. Lateral actuation strain of membrane actuators for silicones materials $\mathbf{A}$ and $\mathbf{B}$ (left) and of selected silicone based materials with increased permittivity (right) as a function of applied electric field.

Table 2. The permittivity, elastic moduli at different strains and the actuation strain at $E_{b}$ for selected materials.

\begin{tabular}{ccccccc}
\hline Entry & $\begin{array}{c}\varepsilon^{\prime} \text { at } \\
10^{4} \mathrm{~Hz}\end{array}$ & $\begin{array}{c}Y_{10 \%}{ }^{\mathrm{a}} \\
{[\mathrm{kPa}]}\end{array}$ & $\begin{array}{c}\varepsilon / Y_{10 \%} \\
{\left[\mathrm{MPa}^{-1}\right]}\end{array}$ & $\begin{array}{c}Y_{100 \%}{ }^{\mathrm{a}} \\
{[\mathrm{kPa}]}\end{array}$ & $\begin{array}{c}E_{b} \\
{[\mathrm{~V} / \mu \mathrm{m}]}\end{array}$ & $\begin{array}{c}S_{l}^{\max } \\
{[\%]}\end{array}$ \\
\hline $\mathbf{A 1}$ & 2.5 & 440 & 5.7 & 130 & 57.5 & 11.6 \\
$\mathbf{A 2}$ & 2.5 & 540 & 4.6 & 148 & 62.4 & 8.7 \\
$\mathbf{B 1}$ & 2.5 & 210 & 11.9 & 102 & 40 & 12.3 \\
$\mathbf{B 2}$ & 2.5 & 130 & 19.2 & 44 & 32.3 & 13.8 \\
$\mathrm{~S}_{\mathrm{PANI}}^{20}$ & 3.5 & 207 & 16.9 & 61 & 43.4 & 15.5 \\
$\mathrm{~S}_{\mathrm{PANI}}^{25}$ & 4 & 116 & 34.5 & 29 & 31.3 & 11.2 \\
$\mathrm{~S}_{\mathrm{CN}}^{100}$ & 10.1 & 152 & 66.4 & 134 & 10.8 & 20.5 \\
$\mathrm{~S}_{\mathrm{H} 62}^{33}$ & 4.5 & 860 & 5.2 & 665 & 56.1 & 8.0 \\
$\mathrm{~S}_{\mathrm{Ag}}^{20}$ & 5.9 & $2.6^{\mathrm{b}}$ & 2.3 & 320 & 13.4 & - \\
$\mathrm{S}_{\mathrm{CF}}^{53}$ & 6 & 15 & 400 & 15 & 7.8 & 5.5 \\
$\mathrm{VHB}^{\mathrm{B}}$ & 4.9 & 176 & 27.8 & 32 & 108.4 & 26.1 \\
\hline
\end{tabular}

${ }^{\mathrm{a}} \mathrm{Y}_{10 \%}$ was determined from the slope of the stress-strain curves using a linear fit to the data point from 0 to $10 \%$ strain while $\mathrm{Y}_{100 \%}$ was determined from the linear fit to the data points within $10 \%$ strain; ${ }^{b}$ the value of $Y$ is given in $\mathrm{MPa}$; 
The electromechanical behavior of the silicone based materials with increased $\varepsilon$ ' is compared to those of softest silicones B and with VHB foil. By far the largest actuation strain was obtained for the VHB which withstands also the highest electric fields. The largest actuation strain at the lowest electric field was obtained for materials $\mathrm{S}_{\mathrm{CN}}^{100}$ with an actuation strain of $20.5 \%$ at $10.8 \mathrm{~V} / \mu \mathrm{m}$. The actuation strain of material $\mathrm{S}_{\mathrm{CF}_{3}}^{53}$ overlaps the one of $\mathrm{S}_{\mathrm{CN}}^{100}$ but its breakdown field is much lower. Thus the larger actuation strain for $\mathrm{S}_{\mathrm{CF}_{3}}^{53}$ was only $5.5 \%$ at $7.8 \mathrm{~V} / \mu \mathrm{m}$. Surprisingly, although the $\varepsilon^{\prime} / Y$ values of $\mathrm{S}_{\mathrm{CN}}^{100}$ are much lower as compared to those of $\mathrm{S}_{\mathrm{CF}_{3}}^{53}$ their actuation strain at a certain voltage was rather similar. For material $\mathrm{S}_{\mathrm{PANI}}^{25}$ and VHB the $\varepsilon^{\prime} / Y$ values are comparable (the $\varepsilon^{\prime} / Y$ values for VHB are slightly higher at large strains) but the actuation strain at a certain electric field for $\mathrm{S}_{\mathrm{PANI}}^{25}$ is much higher. For example $\mathrm{S}_{\mathrm{PANI}}^{25}$ shows a lateral actuation strain of $11 \%$ at $30 \mathrm{~V} / \mu \mathrm{m}$, while VHB shows only $1 \%$ strain at the same electric field. The poor electromechanical sensitivity of VHB might be due to its high viscoelastic losses. Materials $\mathrm{S}_{\mathrm{PANI}}^{20}$ and $\mathrm{S}_{\mathrm{PANI}}^{25}$ have actuation curves that overlap the ones of $\mathbf{B 1}$ and B2, respectively despite of the higher $\varepsilon^{\prime} / Y$ values of $\mathrm{S}_{\mathrm{PANI}}^{\mathrm{x}}$. Although an improvement in the actuation strain was observed when comparing the composites $S_{\mathrm{PANI}}^{\mathrm{x}}$ with the silicone matrix material $\mathbf{B} 1$ used for their synthesis, the improved actuation strain is not that high as one would expect. The poorer actuation response for $\mathrm{S}_{\text {PANI }}^{\mathrm{x}}$ might be due to the viscous or dielectric losses at high electric fields. The actuation strain of material $\mathrm{S}_{\mathrm{H} 62}^{33}$ is slightly higher than the one observed for VHB despite that its $\varepsilon^{\prime} / Y$ values is much lower. The highest electrostatic pressure at breakdown is achieved for VHB while the highest electrostatic pressure at low electric fields $(<10 \mathrm{~V} / \mu \mathrm{m})$ is observed for material $\mathrm{S}_{\mathrm{CN}}^{100}$.

\section{CONCLUSION}

Silicone based materials with tunable mechanical and dielectric properties were presented. The unmodified silicone materials have similar permittivity values $\left(\varepsilon^{\prime}<3\right)$, but different elastic moduli. When tested electromechanically, they show the expected linear increase in the actuation strain with decreasing the elastic moduli. The permittivity of a silicone matrix can be increased by blending it with conductive fillers that are encapsulated in an insulating shell. The encapsulation is important on the one hand to increase the compatibility of the filler with the silicone matrix, and on the other hand to avoid formation of conductive paths through the material. It was observed that when PANI filler was used, materials with slightly higher dielectric breakdowns and significantly higher strain at break as compared to the silicone matrix were obtained. However, the performance of such composites in actuator devices was slightly lower than expected. The reason behind this might rest in the mechanical or dielectric losses of these materials. Elastic composites of $20 \mathrm{vol} \%$ passivated $\mathrm{Ag} / \mathrm{SiO}_{2}$ nanoparticles in a silicone show a moderate increase in the permittivity $\left(\varepsilon^{\prime}=5.9\right.$ at $\sim 1$ $\mathrm{kHz}$ ), an elastic modulus as low as $350 \mathrm{kPa}$ at $100 \%$ strain, and a strain at break of $800 \%$. Despite the rather low values for the dielectric losses at low frequencies $(\tan \delta<0.02)$, the dielectric breakdown was only $13.4 \mathrm{~V} / \mu \mathrm{m}$. The permittivity of a silicone material was significantly increased when modified with polar groups like nitrile. A material with a powerful combination of properties: $\varepsilon^{\prime}>10, Y_{10 \%}=154 \mathrm{kPa}$, and strain at break of $260 \%$ was synthesized. The actuation strain at low electric fields was significantly enhanced. Further work is invested in increasing the dielectric breakdown and the strain at break of these new materials. Given the simplicity of the synthetic protocol used for the nitrile modified silicone elastomer, we expect this material to be scalable and to find its way into applications.

\section{ACKNOWLEDGEMENTS}

We gratefully acknowledge Swiss National Science Foundation (SNF132101, Swiss-Romanian Cooperation Program, grant No. IZERZO_142215/1, and 200021L_140577 / 1), Sciex (12.192), and Swiss Federal Laboratories for Materials Science and Technology (Empa, Dübendorf) for financial support. 


\section{REFERENCES}

[1] Brochu, P., Pei, Q., “Advances in dielectric elastomers for actuators and artificial muscles”, Macromol. Rapid Commun. 31, 10-36 (2010).

[2] Pelrine, R., Kornbluh, R., Pei, Q., Joseph, J., "High-speed electrically actuated elastomers with strain greater than $100 \%$ ", Science, 287, 836-839 (2000).

[3] Carpi, F., De Rossi, D., Kornbluh, R., Perline, R., Sommer-Larsen, P., Dielectric elastomers as electromechanical transducers, Elsevier, Amsterdam, (2008).

[4] Biggs, J., Danielmeier, K., Hitzbleck, J., Krause, J., Kridl, T., Nowak, S., Orselli, E., Quan, X., Schapeler, D., Sutherland, W., Wagner, J., „Electroactive polymers:development of and perspectives for dielectric elastomers” Angew. Chem. Int. Ed. 52, 9409 - 9421 (2013).

[5] Clarson, S. J., Advanced Materials Containing the Siloxane Bond, in Advances in Silicones and SiliconeModified Materials, S. J. Clöarson, ACS Symposium Series; Americam Chemical Society: Washington, DC, (2010); Circu, M., Ko, Y., Nüesch, F. A., Christian, A. G., Opris, D. M., "Soft polydimethylsiloxane thin elastomeric films by in-situ polymerization to be used as dielectricum in actuators", Macromol. Mater. Eng. 299 (9), 1126-1133 (2014).

[6] Stoyanov, H., Brochu, P., Niu, X., Lai, C., Yun, S., Pei, Q., "Long lifetime, fault-tolerant freestanding actuators based on a silicone dielectric elastomer and self-clearing carbon nanotube compliant electrodes", RSC Adv., 3, 2272-2278 (2013).

[7] Goswami, K., Skov, A. L., Daugaard, A. E., "UV-cured, platinum-free, soft polydimethylsiloxane) networks", Chem. Eur. J. 20, 9230 - 9233 (2014).

[8] Molberg, M., Crespy, D., Rupper, P., Nüesch, F., Månson, J.-A. E., Löwe, C., Opris, D. M., "High breakdown field dielectric elastomer actuators using encapsulated polyaniline as high dielectric constant filler", Adv. Funct. Mater. 20, 3280-3291 (2010).

[9] Opris, D. M., Molberg, M., Walder, C., Ko, Y. S., Fischer, B., Nüesch, F. A., "New silicone composites for dielectric elastomer actuator applications in competition with acrylic foil", Adv. Funct. Mater. 21, 3531-3539 (2011).

[10] Pecharroman, C., Moya, J. S. "Experimental evidence of a gigant capacitance in insulator-conductive composites at the percolation threshold", Adv. Mater. 12, 294 (2000).

[11] Quinsaat, J. E. Q., Testino, A., Pin, S., Huthwelker, T., Nuesch, F. A., Bowen, P., Hofmann, H., Ludwig, C., Opris, D. M., "Continuous production of tailored silver nanoparticles by polyol synthesis and reaction yield measured by X-ray absorption spectroscopy: toward a growth mechanism", J. Phys. Chem. $C$, 2014, 118, 11093-11103.

[12] Quinsaat, J. E. Q., Nüesch, F. A., Hofmann, H., Opris, D. M., "Dielectric properties of silver nanoparticles coated with silica shells of different thicknesses", RSC Advances, 3, 6964-6971 (2013).

[13] Quinsaat, J. E. Q., Alexandru, M., Nüesch, F. A., Hofmann, H., Borgschulte, A., Opris, D. M., "Highly stretchable dielectric elastomer composites containing high volume fraction of silver nanoparticles", submitted (2015).

[14] Racles, C., Alexandru, M., Bele, A., Musteata, V. E., Cazacu, M., Opris, D. M., "Chemical modification of polysiloxanes with polar pendant groups by co-hydrosilylation", RSC Adv., 4, 37620-37628 (2014).

[15] Dünki, S. J., Tress, M., Kremer, F., Ko, S. Y., Nüesch, F. A., Racles, C., Opris, D. M., "Fine-tuning of the dielectric properties of polysiloxanes by chemical modification", submitted (2015).

[16] Dünki, S. J., Ko, Y. S., Nüesch, F. A., Opris, D. M., "Self-repairable, high permittivity dielectric elastomers with large actuation strains at low electric fields", submitted (2015). 